\title{
Textbook Outcome
}

\section{Nationwide Analysis of a Novel Quality Measure in Pancreatic Surgery}

\author{
Stijn van Roessel, MD, MSc, ${ }^{*}$ Tara M. Mackay, MD, ${ }^{*}$ Susan van Dieren, MSc, PhD,* \\ George P. van der Schelling, MD, PhD, † Vincent B. Nieuwenhuijs, MD, PhD, $\ddagger$ Koop Bosscha, MD, PhD, $\S$ \\ Edwin van der Harst, MD, Ronald M. van Dam, MD, PhD,\|Mike S. L. Liem, MD, PhD, ${ }^{* *}$ \\ Sebastiaan Festen, MD, PhD, †† Martijn W. J. Stommel, MD, PhD, 㧊Daphne Roos, MD, PhD, §§ \\ Fennie Wit, MD, I. Quintus Molenaar, MD, PhD, ||| Vincent E. de Meijer, MD, PhD, MSc, ${ }^{* * *}$

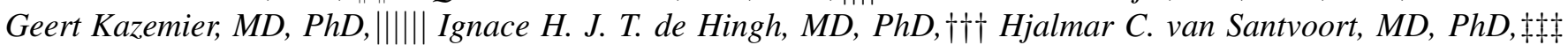 \\ Bert A. Bonsing, MD, PhD, §§§ Olivier R. Busch, MD, PhD, ${ }^{*}$ Bas Groot Koerkamp, MD, PhD, MSc, $\uparrow ণ$ \\ and Marc G. Besselink, MD, PhD, MSc ${ }^{*}$, for the Dutch Pancreatic Cancer Group
}

\begin{abstract}
Background: Textbook outcome (TO) is a multidimensional measure for quality assurance, reflecting the "ideal" surgical outcome.

Methods: Post-hoc analysis of patients who underwent pancreatoduodenectomy (PD) or distal pancreatectomy (DP) for all indications between 2014 and 2017, queried from the nationwide prospective Dutch Pancreatic Cancer Audit. An international survey was conducted among 24 experts from 10 countries to reach consensus on the requirements for TO in pancreatic surgery. Univariable and multivariable logistic regression was performed to identify TO predictors. Between-hospital variation in TO rates was compared using observed-versus-expected rates.
\end{abstract}

Results: Based on the survey ( $92 \%$ response rate), TO was defined by the absence of postoperative pancreatic fistula, bile leak, postpancreatectomy hemorrhage (all ISGPS grade $\mathrm{B} / \mathrm{C}$ ), severe complications (Clavien-Dindo $\geq$ III), readmission, and in-hospital mortality. Overall, 3341 patients were included (2633 (79\%) PD and 708 (21\%) DP) of whom 60.3\% achieved TO; $58.3 \%$ for PD and $67.4 \%$ for DP. On multivariable analysis, ASA class 3 predicted a worse TO rate after PD (ASA 3 OR 0.59 [0.44-0.80]), whereas a dilated pancreatic duct $(>3 \mathrm{~mm})$ and pancreatic ductal adenocarcinoma

From the *Department of Surgery, Cancer Center Amsterdam, Amsterdam UMC, University of Amsterdam, Amsterdam, The Netherlands; †Department of

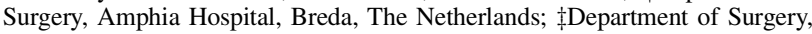
Isala Clinics, Zwolle, The Netherlands; §Department of Surgery, Jeroen Bosch Hospital, 's Hertogenbosch, The Netherlands; - Department of Surgery, Maasstad Hospital, Rotterdam, The Netherlands; ||Department of Surgery, Maastricht University Medical Center, Maastricht, The Netherlands; **Department of Surgery, Medisch Spectrum Twente, Enschede, The Netherlands; ††Department of Surgery, OLVG, Amsterdam, The Netherlands; ł†Department of Surgery, Radboud University Medical Center, Nijmegen, The Netherlands; §§Department of Surgery, Reinier de Graaf ziekenhuis, Delft, The Netherlands; $\uparrow$ Department of Surgery, Tjongerschans, Heerenveen, The Netherlands; ||||Department of Surgery, University Medical Center Utrecht, Utrecht, The Netherlands; ${ }^{* *}$ Department of Surgery, University Medical Center Groningen, Groningen, The Netherlands; †† †Department of Surgery, Catharina Hospital, Eindhoven, The Netherlands; † †Department of Surgery, St. Antonius

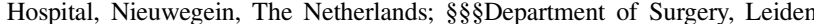
University Medical Center, Leiden, The Netherlands; $\uparrow$ Department of Surgery, Erasmus MC University Medical Center, Rotterdam, The Netherlands; and ||||||Department of Surgery, Cancer Center Amsterdam, Amsterdam UMC, Vrije Universiteit Amsterdam, Amsterdam, The Netherlands.

凶m.g.besselink@amc.nl.

SvR and TMM share first authorship.

The author reports no conflicts of interest.

Supplemental digital content is available for this article. Direct URL citations appear in the printed text and are provided in the HTML and PDF versions of this article on the journal's Web site (www.annalsofsurgery.com).

Copyright (C) 2019 Wolters Kluwer Health, Inc. All rights reserved.

ISSN: 0003-4932/19/27101-0155

DOI: $10.1097 /$ SLA.0000000000003451
(PDAC) were associated with a better TO rate (OR 2.22 [2.05-3.57] and OR 1.36 [1.14-1.63], respectively). For DP, female sex and the absence of neoadjuvant therapy predicted better TO rates (OR 1.38 [1.01-1.90] and OR 2.53 [1.20-5.31], respectively). When comparing institutions, the observedversus-expected rate for achieving TO varied from 0.71 to 1.46 per hospital after casemix-adjustment.

Conclusions: TO is a novel quality measure in pancreatic surgery. TO varies considerably between pancreatic centers, demonstrating the potential benefit of quality assurance programs.

Keywords: auditing, outcomes, pancreatic surgery, practice variation, surgery, textbook outcome

(Ann Surg 2020;271:155-162)

linical auditing and quality improvement is increasingly important in the field of surgery. ${ }^{1}$ This process is also driven by hospitals, governmental institutions, and other providers as they increasingly demand transparency to monitor and improve clinical outcomes. $^{2}$ In the United States and Europe, several prospective surgical registries have been established to support this goal. ${ }^{3-6}$ In the Netherlands, clinical auditing designed for quality improvement purposes and reduction of unwanted variation in clinical practice has been implemented for many surgical procedures. ${ }^{7}$ One of these audits is the Dutch Pancreatic Cancer Audit (DPCA), a mandatory nationwide prospective registry for pancreatic surgery. ${ }^{6}$

Monitoring and comparing quality of specific procedures, such as pancreatic resections, requires a broad judgement which may be difficult to obtain. ${ }^{8,9}$ Although different outcome parameters are generally related to each other, a composite outcome measure based on a variety of short-term outcomes might offer a better, and potentially more stable reflection of overall quality than singleoutcome indicators like mortality, morbidity, or readmission rates. ${ }^{10,11}$ Textbook Outcome (TO) is such a composite outcome, which attempts to cover the entire surgical process in a single indicator. TO, reflecting the ideal surgical outcome, is realized when all prespecified requirements of TO are fulfilled according to an allor-none principle. ${ }^{12}$ TO has been defined for several surgical procedures, including abdominal aneurysm surgery, oesophageal/gastric surgery, and colorectal surgery. ${ }^{13-15}$ The requirements of TO would ideally be disease-specific and surgery-related parameters, covering all important aspects of a patients' postoperative course with the possibility for "case-mix correction" to account for variance in patient populations between centers. The definition of surgical quality and what constitutes TO, however, is arbitrary, subject to 
cultural differences and might even change over time. ${ }^{16}$ Nevertheless, previous studies in other surgical fields demonstrated that TO was a feasible and useful parameter for comparison of quality between institutions, also showing correlation with improved long-term outcomes after cancer surgery. ${ }^{13-15,17,18}$

This study proposes a TO definition for pancreatic surgery, based on the opinion of international experts, and assesses predictors and associations with TO in a nationwide surgical audit.

\section{METHODS}

\section{Study Design and Data Collection}

Patients who underwent pancreatoduodenectomy (PD) or distal pancreatectomy (DP) for all indications between January 2014 and December 2017 in the Netherlands were eligible for this study. Data were obtained from the prospectively maintained, mandatory, nationwide DPCA. ${ }^{6}$ The DPCA covers all pancreatic resections for both malignant and benign/premalignant tumors in the Netherlands since January 2014. A previously published internal audit of the DPCA demonstrated a high degree of accuracy $(97.2 \%)$ and case ascertainment $(93.0 \%){ }^{6}$ Patients with missing data on 1 or more of the requirements for TO according to the survey were excluded.

Baseline characteristics, pathological parameters, and shortterm outcomes up until 30 days postdischarge are collected by each institution and are centrally archived in a nationwide database. Study proposals and data requests using the DPCA are reviewed by a scientific board of the Dutch Pancreatic Cancer Group, after which the data, in case of unanimous approval, are anonymized and provided for research purposes. As anonymized data was used, no ethical approval was required by the Institutional Review Boards.

Regarding pathology definitions, any type of ductal adenocarcinoma (pancreatic, ampullary, distal bile duct, or duodenal), and neuro-endocrine tumors grade II and III of the pancreas were classified as malignant. ${ }^{19}$ Noninvasive IPMN, neuroendocrine tumors grade I, and solid pseudopapillary neoplasms were classified as premalignant/benign.

\section{Survey on the Definition of Textbook Outcome}

In order to reach international consensus on all requirements for TO, a survey was conducted among 24 internationally recognized experts from 21 institutions, in 10 different countries spanning 4 continents. Experts were systematically identified by contacting the senior authors of large single-center studies ( $>1000$ PDs) or studies on quality improvement in (pancreatic) surgery. The survey consisted of 17 items, which were each rated on a 5-point Likert scale whether the respondent agreed on that particular item to be a requirement for TO. An agreement rate of $>80 \%$ per item was considered as consensus and resulted in the item being included in the definition of TO. The survey outcome and definition of TO are presented in the Results section. The international experts are presented in the supplementary table S2, http://links.lww.com/ SLA/B689.

\section{Statistical Analysis}

Continuous variables were presented as median with interquartile range (IQR), or dichotomized on a clinically relevant cut-off value. Categorical variables were presented as frequencies and proportions, or in some cases as proportions only to omit large numbers. TO was determined for each patient, according to the selected items through the survey. TO was achieved when a patients' admission met all TO requirements. TO rates were compared between groups using the Chi-square test.
Multiple imputation was used to impute missing baseline and treatment variables by creating 5 datasets, using the mice package in $\mathrm{R}$ (predictive mean matching). In order to assess between-hospital variations in TO rate, casemix-adjustment was performed with logistic regression using relevant baseline variables which may have played a role in patient selection (sex, age [continuous], BMI [continuous], preoperative low serum albumin [below $3.5 \mathrm{~g} / \mathrm{dL}$ ], WHO performance status $[0,1,2$, or 3$]$, pancreatic texture [soft or hard], and pathology [pancreatic cancer or other]). Based on the covariates (ie, casemix) a predicted probability to achieve TO was estimated, with subsequently observed-versus-expected $(\mathrm{O} / \mathrm{E}) \mathrm{TO}$ rates being calculated per hospital. $\mathrm{O} / \mathrm{E}$ rates higher than 1 represent better TO rates than predicted and O/E rates lower than 1 worse than predicted, based on the casemix for that specific hospital. $\mathrm{O} / \mathrm{E}$ rates were presented in a funnel plot to visualize volume-outcome relationships. Additionally, multivariable logistic regression was performed to identify clinicopathological predictors of TO. Variables were excluded through backward selection until only statistically significant variables were selected in the final model. Two-sided $P$ values of less than 0.05 were considered statistically significant and odds ratios (OR) and their 95\% confidence intervals (CI) were reported. All analyses were performed using $\mathrm{R}$ version 3.4.3 (cran.r-project.org).

\section{RESULTS}

\section{Survey Outcome}

Based on the survey (92\% response rate), 6 out of the 17 items were selected as requirements for TO with an agreement rate of $>80 \%$. TO was defined as the absence of postoperative pancreatic fistula (POPF), bile leak, postpancreatectomy hemorrhage (all grade $\mathrm{B} / \mathrm{C}$ according to ISGPS or ISGLS), ${ }^{20-22}$ severe complications (Clavien-Dindo grade $\geq \mathrm{III}$ ), ${ }^{23}$ readmission within 30 days after discharge, and in-hospital or 30-day mortality. For the current study only in-hospital mortality was available in the DPCA, which was used instead in our analyses. Furthermore, the items regarding complications rated as Clavien-Dindo grade $\geq$ III and a postoperative reintervention (surgical, endoscopic, or radiological) were combined in 1 item, due to the same nature of these items. Since previous TO publications also included prolonged length of stay (LOS) as requirement, additional analyses were performed using an extended definition (TO+LOS) with prolonged LOS defined as $>14$ days. The various agreement rates of the survey are presented in the supplementary tables S1 and S2, http://links.lww.com/SLA/B689.

\section{Baseline Characteristics}

In total, 3520 patients met the inclusion criteria. Of those, 179 $(5.1 \%)$ patients were excluded for missing data on 1 or more requirements of TO. The final cohort consisted of 3341 patients, of which 2633 (78.8\%) patients underwent PD and $708(21.2 \%)$ patients underwent DP. The median age was 67 (IQR 58-74) years, $54.3 \%$ of the patients were male. The majority of patients had an American Society of Anesthesiologists (ASA) score of 1 or 2 $(77.8 \%)$, a WHO performance status of 0 or $1(89.0 \%)$, a BMI lower than $30 \mathrm{~kg} / \mathrm{m}^{2}(86.6 \%)$ and underwent surgery for a malignancy (76.6\%). In-hospital mortality was $3.2 \% ; 3.7 \%$ for PD and $1.5 \%$ for DP, respectively $(P=0.006)$. All baseline characteristics are presented in Table 1.

\section{Textbook Outcome Rates}

The overall proportion of patients who achieved TO was $60.3 \%$. TO was realized in $58.3 \%$ of patients after PD versus $67.4 \%$ of patients after DP $(P<0.001)$. Among the different items of TO, a complication of Clavien-Dindo grade $\geq$ III (requiring 
TABLE 1. Baseline Characteristics by Type of Surgery

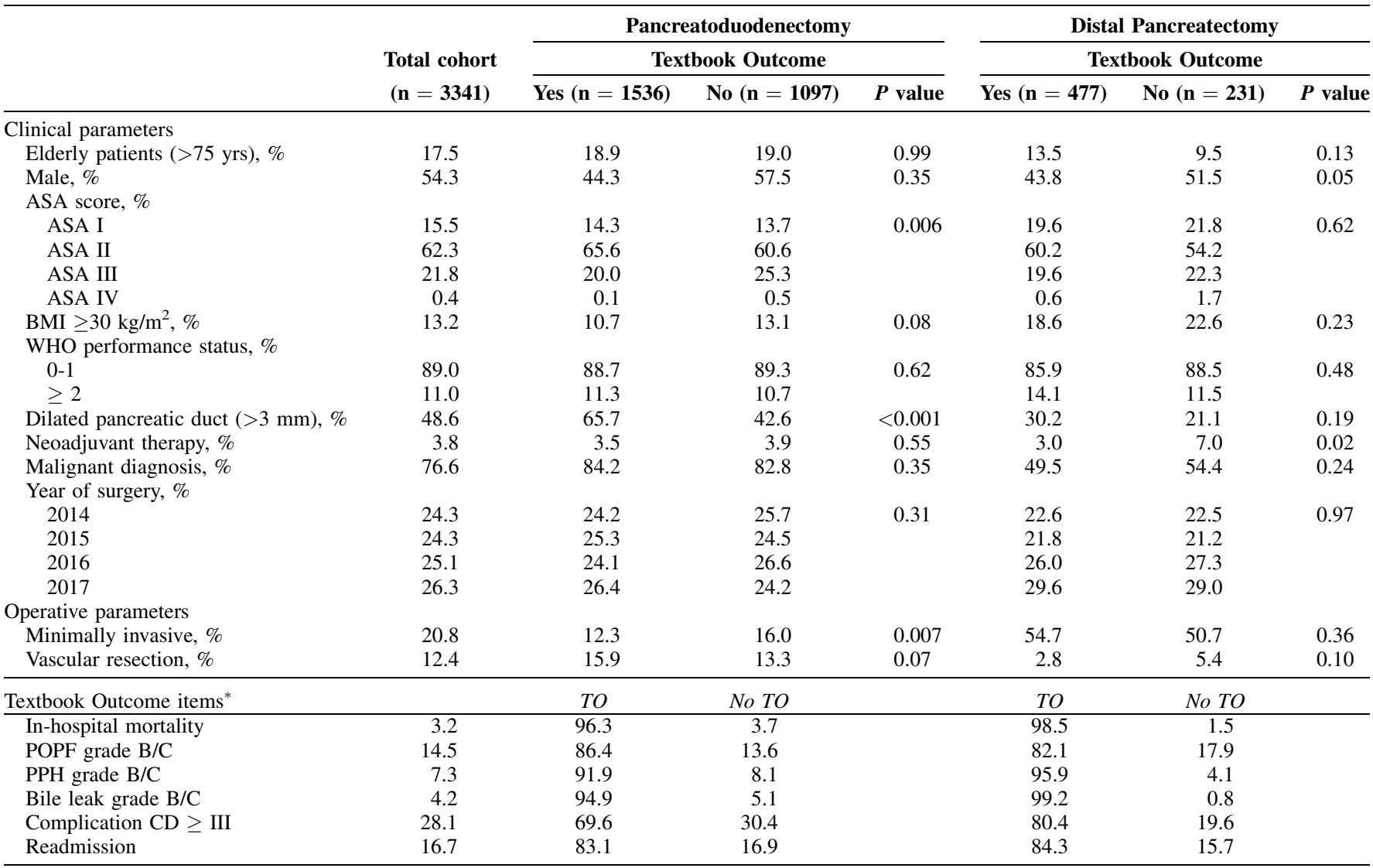

${ }^{*}$ Textbook outcome percentages are displayed per item, TO versus no TO.

Values are percentages. ASA indicates American Association of Anesthesiologists; BMI, body mass index; PPPD, pylorus-preserving pancreatoduodenectomy; WHO, World Health Organization.

endoscopic, radiologic, or surgical reintervention) occurred most often with a rate of $30.4 \%$ and $19.6 \%$ for patients who underwent PD and DP, respectively $(P<0.001)$. The rates of the 6 TO items and the cumulative percentages are displayed in Figure 1. Among patients who underwent PD, patients with TO had lower ASA scores $(P=$ $0.006)$ and more often a dilated pancreatic duct $(P<0.001)$ than patients who did not achieve TO. Among patients who underwent DP, patients with TO less often received neoadjuvant therapy $(P=0.02)$.

\section{Between-hospital Variation}

During 2014 to 2017, 20 centers performed pancreatic surgery in the Netherlands, with 18 centers performing $\geq 20$ PDs annually and, of those, 5 centers performing $\geq 40$ PDs annually. The median number of annually performed procedures was 33 (IQR 27-39) for PD and 8 (IQR 5-10) for DP per hospital. Unadjusted TO rates varied considerably between hospitals, with a range of $35.2 \%$ to $87.7 \%$ for PD and $47.6 \%$ to $91.2 \%$ for DP. When adjusted for casemix, the $\mathrm{O} / \mathrm{R}$ rates ranged from 0.71 to 1.46 per hospital for all pancreatic surgeries, with $\mathrm{O} / \mathrm{R}$ rates ranging from 0.61 to 1.56 for PD and from 0.71 to 1.32 for DP. There was no evident correlation between procedure volume and TO rate, as presented in Figure $2 \mathrm{~A}$ and $\mathrm{B}$.

\section{Multivariable Analysis}

On multivariable analysis, only class ASA 3 predicted a worse TO rate after PD (OR 0.62 [0.46-0.83]), whereas a dilated pancreatic duct $(>3 \mathrm{~mm})$ and pancreatic ductal adenocarcinoma (PDAC) were associated with a better TO rate (OR 2.22 [2.05-3.57] and OR 1.36 [1.14-1.63], respectively). For DP, female sex and the absence of neoadjuvant therapy were associated with a better TO rate (OR 1.38 [1.01-1.90] and OR 2.53 [1.20-5.31], respectively). Results of univariable and multivariable analysis are presented for PD and DP in Tables 2 and 3, respectively.

\section{Additional Analyses Using the Extended Definition (TO+LOS)}

When applying the extended definition, TO+LOS rates decreased from 58.3 to $47.5 \%$ for PD and from 67.4 to $63.3 \%$ for DP. When assessing factors associated with TO+LOS on multivariate analysis, age $>75$ years was identified as additional risk factor for a lower TO+LOS rate after PD (OR 0.78 [95\% CI 0.64-0.96]). Among patients who underwent DP, vascular resection was identified as additional predictor for TO+LOS (OR 0.34 [95\% CI 0.14-0.79]). The rest of the results did not change significantly with the extended definition.

\section{DISCUSSION}

This study proposed a novel consensus-based definition of TO in pancreatic surgery (absence of all of the following parameters: postoperative pancreatic fistula, bile leak, postpancreatectomy hemorrhage, Clavien-Dindo $\geq$ III complications, readmission and in- 
The different items of Textbook Outcome

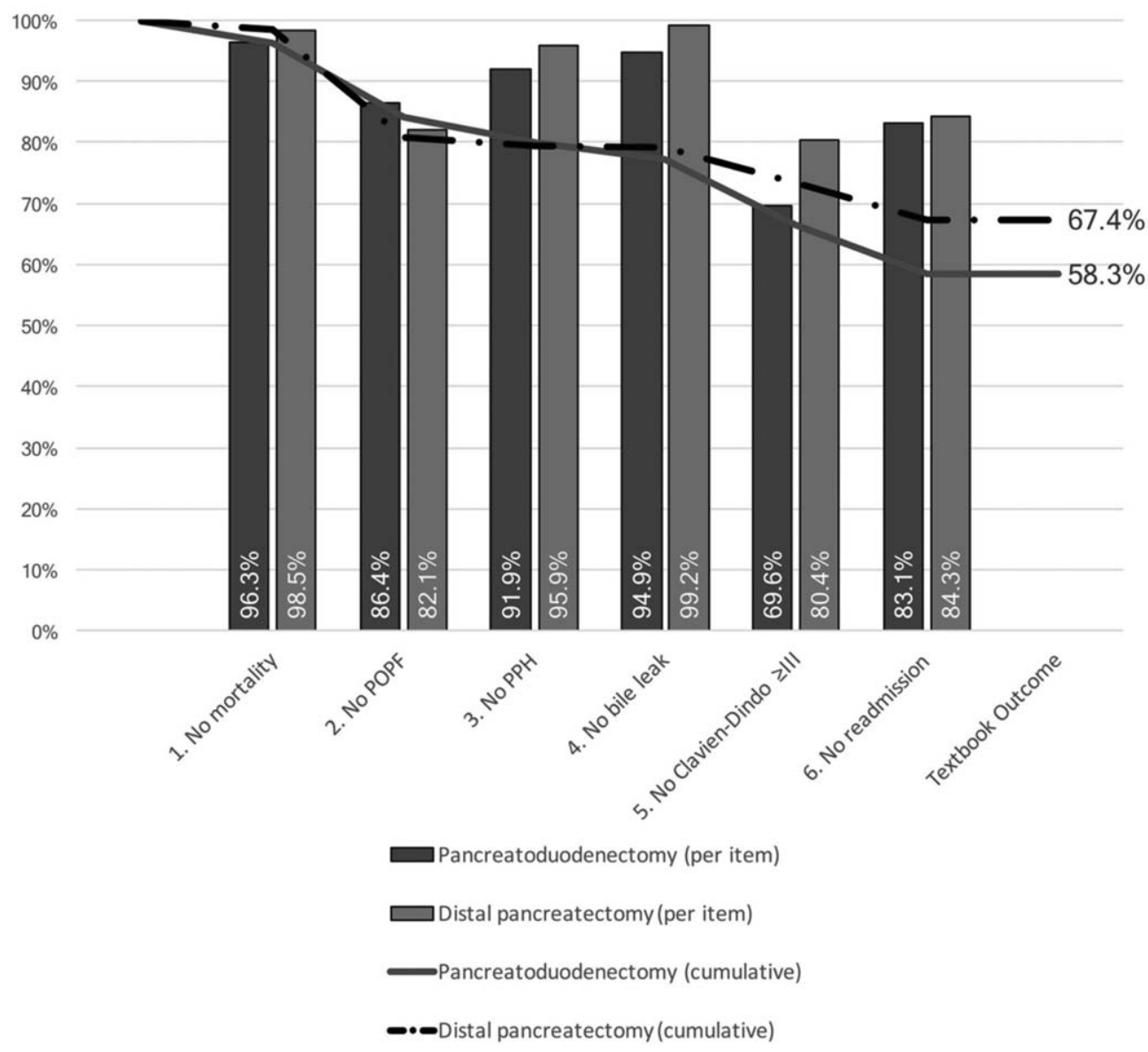

FIGURE 1. Textbook Outcome percentages (per item and cumulative) for pancreatoduodenectomy and distal pancreatectomy.

hospital mortality) as agreed by an international survey. The nationwide analysis demonstrated its feasibility and utility to assess clinical outcome of pancreatic surgery with $60.3 \%$ TO. On multivariable analysis, a higher ASA class was predictive for a worse TO rate after $\mathrm{PD}$, whereas a dilated pancreatic duct $(>3 \mathrm{~mm})$ and PDAC were associated with an improved TO rate. For DP, female sex and the absence of neoadjuvant therapy were independently associated with improved TO rates. There was considerable variation in TO rates between pancreatic centers, supporting the need and potential for quality assurance programs. The present study demonstrated that only limited preoperative available parameters were predictive for TO. On a nationwide basis, however, TO might be a powerful outcome measure to be evaluated as an instrument for comparing quality of surgical practice between different institutions and national registries.

The multidimensional parameter TO is a relatively new concept which was found useful in other fields of surgery. ${ }^{13-}$ $15,17,18$ Our definition of TO was established by an international survey, conducted among experts in quality improvement in (pancreatic) surgery. Out of 17 items, absence of 6 complications was considered to reflect the ideal outcome after pancreatic surgery. Prolonged hospital stay was initially not included in these 6 requirements. Although hospital readmissions are generally expensive, at times avoidable and in some countries even a tool for penalization, ${ }^{24,25}$ many cultural differences exist worldwide regarding hospital stay. For instance, in southern Europe and in Japan patients are often not discharged until fully recovered, which may take 3 to 4 weeks. The external validity of TO may therefore actually benefit by not including prolonged hospital stay as a complication. Recently, another study investigated TO among Medicare patients who underwent hepatopancreatic surgery. ${ }^{26}$ This study included length of stay as one of the requirements for TO $(>50$ th percentile for each procedure). This study also combined distal pancreatectomy and pancreateoduodenectomy, impeding the generalizability and translatability of the results to daily practice. The variation in overall TO rates in this study [range $11.1-69.6 \%$ ] seems fairly in line with our findings. ${ }^{26}$

Compared with the TO definition in colorectal and oesophagogastric surgery, the proposed TO definition for pancreatic surgery does not contain pathology-related variables. ${ }^{14,15}$ This decision was taken after consultation with several pancreatic pathologists for 2 reasons. First, pancreatic surgery includes a considerable proportion of benign and premalignant lesions (23.4\% in the present study). With these patients in mind, incorporating resection margin status and lymph node yield would further complicate the definition of TO. Second, the R1 rate in pancreatic surgery does not necessarily reflect surgical technique but rather the extent of pathological assessment and the disease itself. ${ }^{27,28}$ For example, tumor invasion in the anterior 


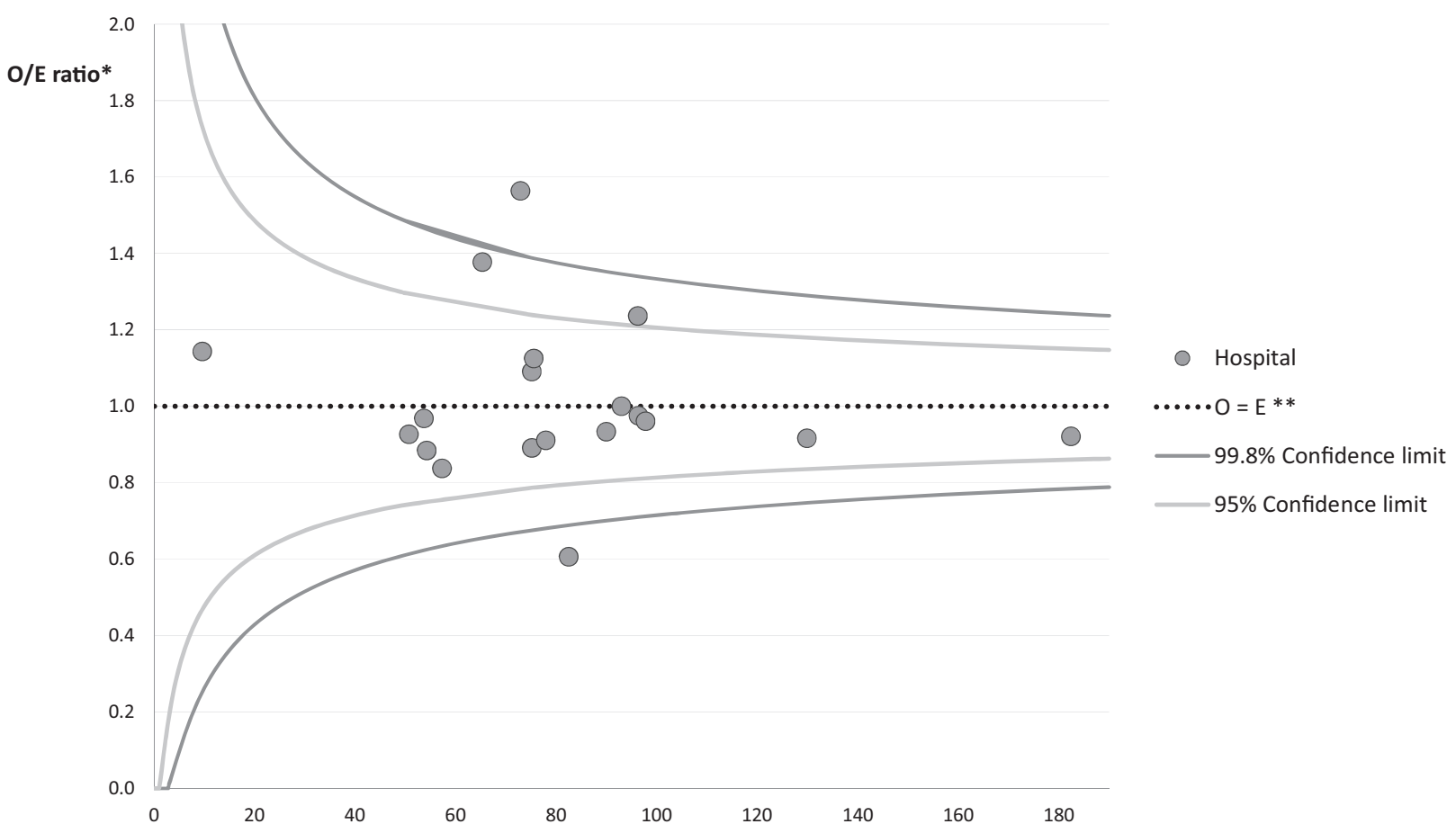

A

Expected^ $^{\wedge}$ number of patients with Textbook Outcome

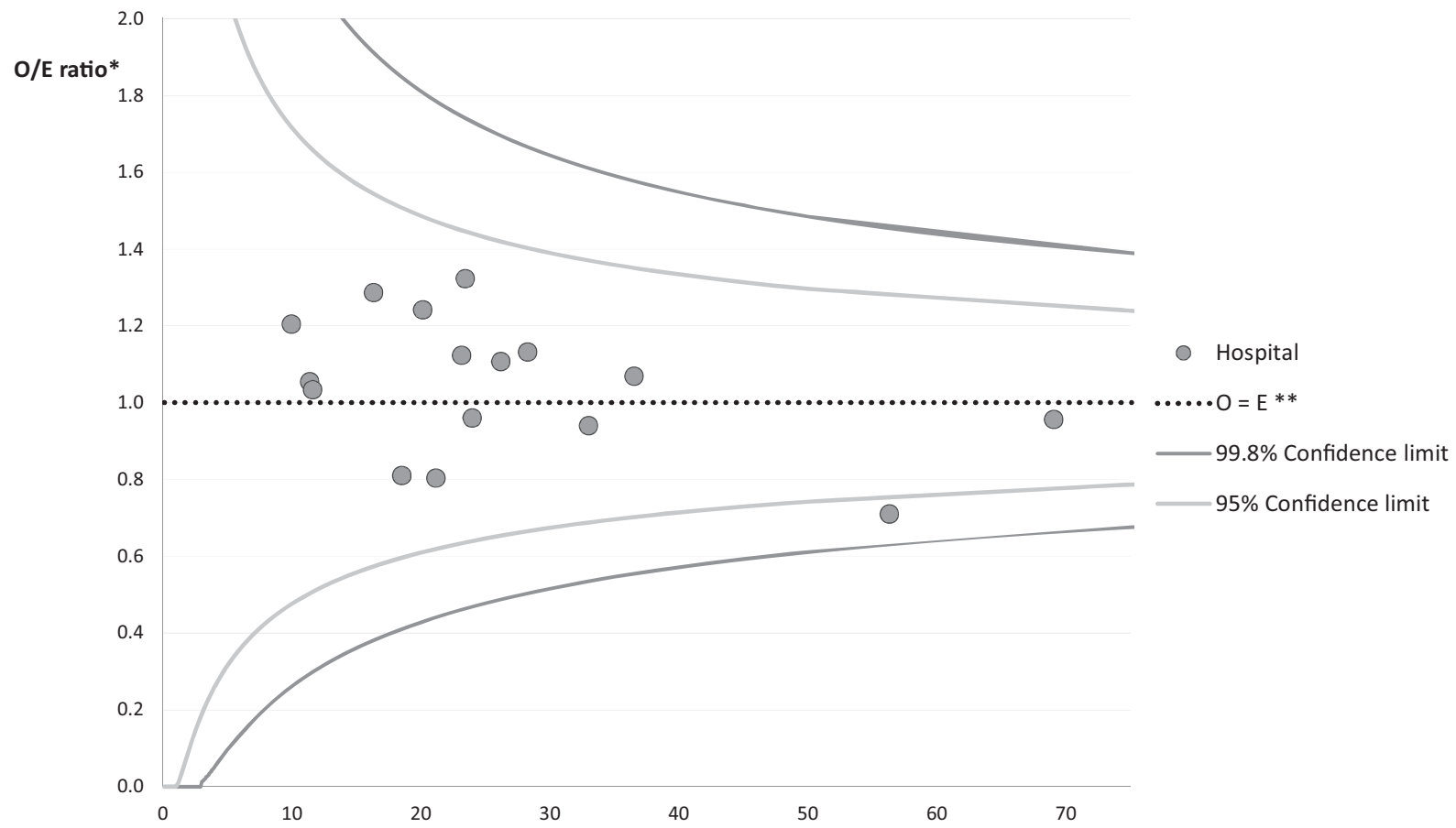

B

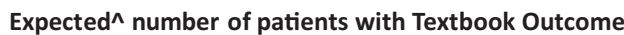

FIGURE 2. A, Funnel plot of between-hospital variation in Textbook Outcome after pancreatoduodenectomy during 2014-2017. ${ }^{*} \mathrm{O} / \mathrm{E}$ ratio: observed number of Textbook Outcome patients divided by expected ${ }^{\wedge}$ number of Textbook Outcome patients. ${ }^{* *} \mathrm{O}=\mathrm{E}$ : the observed number equals the expected ${ }^{\wedge}$ number of Textbook Outcome patients. ${ }^{\wedge}$ Expected based on population characteristics in t. B, Funnel-plot of between-hospital variation in Textbook Outcome after distal pancreatectomy during 2014-2017. ${ }^{*} \mathrm{O} / \mathrm{E}$ ratio: observed number of Textbook Outcome patients divided by expected ${ }^{\wedge}$ number of Textbook Outcome patients. ${ }^{* *} \mathrm{O}=\mathrm{E}$ : the observed number equals the expected ${ }^{\wedge}$ number of Textbook Outcome patients. ${ }^{\wedge}$ Expected based on population characteristics in $t$. 
TABLE 2. Multivariate Analysis of Parameters Associated With Textbook Outcome After Pancreatoduodenectomy

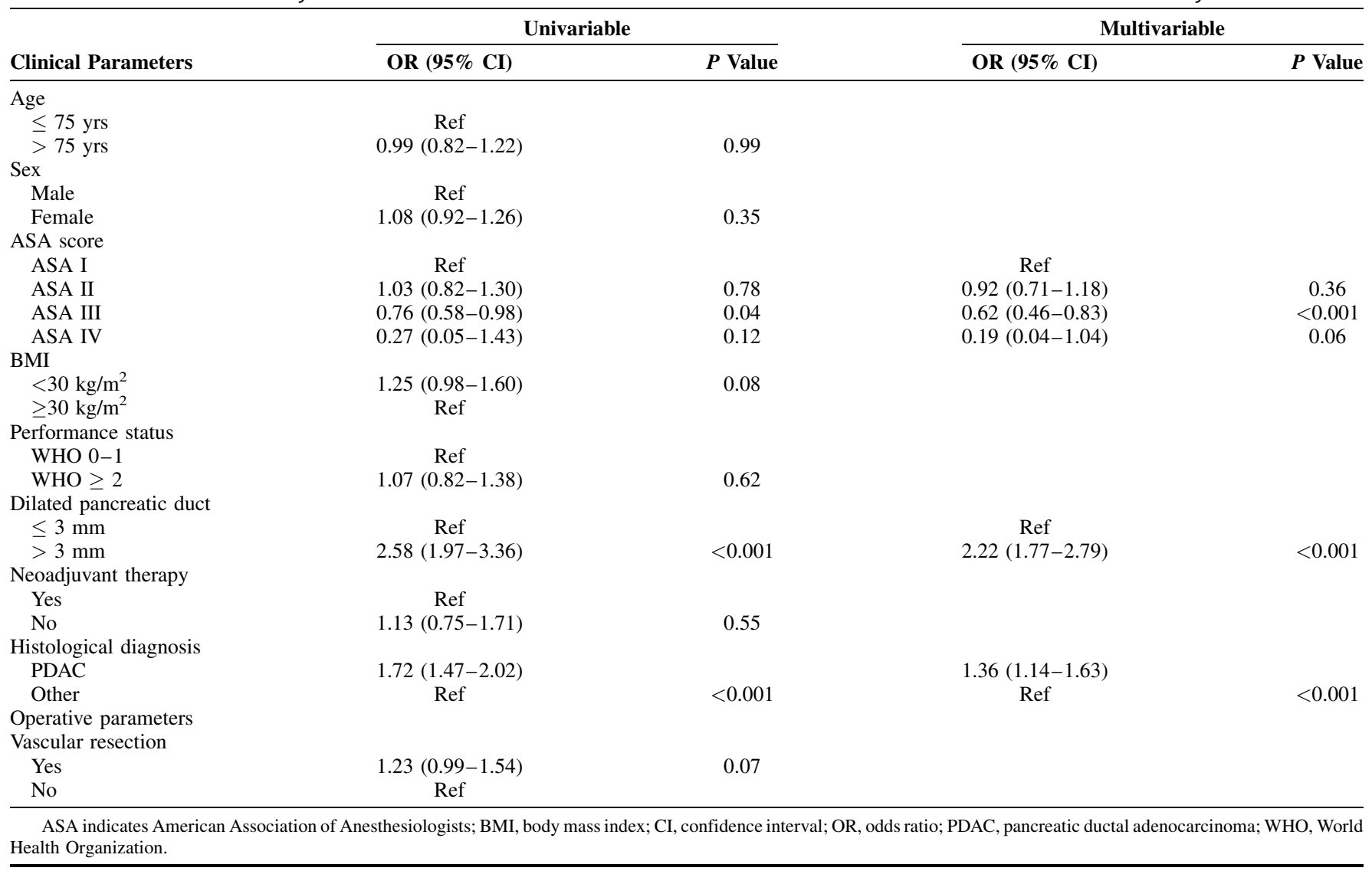

margin results in an $\mathrm{R} 1$ resection, which is not under influence of the surgeon and more a sign of advanced disease. Therefore, only surgical and no pathological variables were included in our TO definition for pancreatic surgery.

Other variables related to the quality of pancreatic surgery could also be considered, such as prolonged operative time, intraoperative blood loss, or postoperative transfusions. Although these variables have been found to be associated with worse outcomes, ${ }^{29,30}$ it might be difficult to obtain accurate measurements and these parameters might not necessarily imply lower surgical quality but rather more difficult cases. More importantly, the experts of the TO design panel decided not to include intraoperative parameters in the survey round, so eventually only items related to postoperative clinical outcome were considered most relevant to the patient.

In the literature, several factors have been identified as predictors for postoperative morbidity and mortality after PD, including a higher ASA score, a small pancreatic duct, a soft pancreas, high-risk pathology (nonmalignant), and excessive blood loss. ${ }^{31-33}$ Our results showed that patients with a higher ASA class, a smaller pancreatic duct $(<3 \mathrm{~mm})$ and a diagnosis other than PDAC were independently associated with lower TO rates after PD, which are in line with previously published findings. For DP, similar risk factors have been shown to be associated with POPF after DP, including a soft pancreas, neuroendocrine or nonmalignant pathology, a higher BMI, blood transfusion, and longer operative time. ${ }^{34,35}$ Remarkably, our findings demonstrate superior TO rates in female patients and those who did not receive neoadjuvant treatment.
Perhaps, patients who were selected for neoadjuvant therapy were more likely to undergo extensive surgery with multivisceral resection due to more advanced tumors. Not enough covariates (ie, potential confounders) were available to adjust for this relationship and to warrant causal inference.

Our study also identified a considerable variability of TO rates between pancreatic centers, ranging from 35.2 to $87.7 \%$ for PD and 47.6 to $91.2 \%$ for DP. Although subject to sampling error and potentially selection bias, especially for lower volume centers, this variability would seem undesirable. Quality assurance programs could improve this variation. Casemix-adjustment did not reduce much of the differences in TO rates between institutions. This minimal effect of casemix-adjustment suggests that patient selection played a minor role in the observed differences between centers. In addition to assessing predictors or factors associated with TO, the key in assessing surgical quality through parameters such as TO is mainly in revealing practice variation and potentially identify institutions that might benefit most from quality improvement. Besides improving treatments at already excellent institutions, quality improvement programs might help to improve overall and nationwide performance. In evaluating surgical quality in a broad perspective, TO demonstrates to be a useful indicator.

These results should be interpreted with several limitations in mind. First, in a nationwide registry not all parameters are recorded, or not recorded with sufficient detail. This could preclude in-depth study of relationships and adjustment for all potential confounders. Second, there remains significant variation in postoperative strategy between 
TABLE 3. Multivariate Analysis of Parameters Associated With Textbook Outcome After Distal Pancreatectomy

\begin{tabular}{|c|c|c|c|c|}
\hline \multirow[b]{2}{*}{ Clinical Parameters } & \multicolumn{2}{|c|}{ Univariable } & \multicolumn{2}{|c|}{ Multivariable } \\
\hline & OR $(95 \%$ CI $)$ & $P$ Value & OR $(95 \%$ CI $)$ & $P$ Value \\
\hline \multicolumn{5}{|l|}{ Age } \\
\hline$\leq 75 \mathrm{yrs}$ & Ref & & & \\
\hline \multicolumn{5}{|l|}{ Sex } \\
\hline Male & Ref & & Ref & \\
\hline Female & $1.36(0.99-1.87)$ & 0.05 & $1.38(1.01-1.90)$ & 0.05 \\
\hline ASA II & $1.23(0.82-1.85)$ & 0.31 & & \\
\hline ASA III & $0.98(0.60-1.59)$ & 0.93 & & \\
\hline ASA IV & $0.40(0.09-1.87)$ & 0.25 & & \\
\hline \multicolumn{5}{|l|}{ BMI } \\
\hline$<30 \mathrm{~kg} / \mathrm{m}^{2}$ & $1.28(0.86-1.90)$ & 0.23 & & \\
\hline$\geq 30 \mathrm{~kg} / \mathrm{m}^{2}$ & Ref & & & \\
\hline \multicolumn{5}{|l|}{ Performance status } \\
\hline$>3 \mathrm{~mm}$ & $1.63(0.81-3.28)$ & 0.19 & & \\
\hline \multicolumn{5}{|l|}{ Neoadjuvant therapy } \\
\hline Yes & Ref & & Ref & \\
\hline No & $2.46(1.17-5.14)$ & 0.02 & $2.53(1.20-5.31)$ & 0.01 \\
\hline \multicolumn{5}{|l|}{ Histological diagnosis } \\
\hline PDAC & $0.79(0.56-1.12)$ & 0.19 & & \\
\hline Other & Ref & & & \\
\hline \multicolumn{5}{|l|}{ Operative parameters } \\
\hline \multicolumn{5}{|l|}{ Vascular resection } \\
\hline Yes & $0.52(0.23-1.16)$ & 0.11 & & \\
\hline No & Ref & & & \\
\hline
\end{tabular}

institutions, that is, percutaneous catheter drainage for small amounts of peripancreatic fluid after pancreatoduodenectomy or endoscopic placement of a nasojejunal feeding tube (both considered as a Clavien-Dindo grade III complication and thus, consequently, absence of TO). ${ }^{23}$ However, adequate complication management also reflects hospital experience and expertise, which consequently has its impact on clinical outcome. ${ }^{36}$ Third, it should be noted that TO does not make a distinction in terms of severity. Patients with a Clavien-Dindo grade III complication and otherwise uncomplicated course have the same score (no TO) as a postoperative death. Fourth, TO is an important outcome parameter as experienced by involved surgeon and not by involved patients. In this sense patient recorded outcome measures (ie, discharge to home or revalidation clinic, ability to work) will gain significance in future quality programs. Furthermore, it should be addressed that if TO would be implemented as a part of a nationwide audit, defensive strategies, like strict patient selection in certain centers, could emerge. This emphasizes that TO should always be used in addition to the other quality indicators.

Nevertheless, TO is a promising multidimensional indicator. It reflects quality better than a single indicator and is suitable to guide quality improvement. Although it remains difficult to predict TO preoperatively, it may offer a powerful parameter to assess between-hospital variation and to compare quality between institutions or audits. In other surgical audits in the Netherlands, TO has already been successfully implemented as quality indicator for internal auditing purposes. Hospitals are able to see their casemix-adjusted TO rate in relation to the TO rates of other institutions, which might lead to an intrinsic drive for quality assurance.

\section{REFERENCES}

1. Bilimoria KY, Bentrem DJ, Lillemoe KD, et al. Assessment of pancreatic cancer care in the United States based on formally developed quality indicators. J Natl Cancer Inst. 2009;101:848-859.

2. Wouters MW, Jansen-Landheer ML, van de Velde CJ. The Quality of Cancer Care initiative in the Netherlands. Eur J Surg Oncol. 2010;36(suppl 1):S3-s13.

3. Wellner UF, Keck T. Quality indicators in pancreatic surgery: lessons learned from the German DGAV StuDoQ|Pancreas Registry. Visceral Med. 2017;33:126-130.

4. Beane JD, House MG, Ceppa EP, et al. Variation in drain management after pancreatoduodenectomy: early versus delayed removal. Ann Surg. 2019;269:718-724.

5. Tingstedt B, Andersson B, Jonsson C, et al. First results from the Swedish National Pancreatic and Periampullary Cancer Registry. HPB (Oxford). 2019;21:34-42.

6. van Rijssen LB, Koerkamp BG, Zwart MJ, et al. Nationwide prospective audit of pancreatic surgery: design, accuracy, and outcomes of the Dutch Pancreatic Cancer Audit. HPB (Oxford). 2017;19:919-926.

7. Coebergh van den Braak RRJ, van Rijssen LB, van Kleef JJ, et al. Nationwide comprehensive gastro-intestinal cancer cohorts: the 3P initiative. Acta Oncol. 2018;57:195-202.

8. Donabedian A. Evaluating the quality of medical care. Milbank Quart. 2005;83:691-729.

9. Donabedian A. The quality of care. How can it be assessed? JAMA 1988;260:1743-1748

10. Nimptsch U, Krautz C, Weber GF, et al. Nationwide in-hospital mortality following pancreatic surgery in Germany is higher than anticipated. Ann Surg. 2016;264:1082-1090.

11. Tsai TC, Joynt KE, Orav EJ, et al. Variation in surgical-readmission rates and quality of hospital care. N Engl J Med. 2013;369:1134-1142.

12. Nolan T, Berwick DM. All-or-none measurement raises the bar on performance. JAMA. 2006;295:1168-1170. 
13. Karthaus EG, Lijftogt N, Busweiler LAD, et al. Textbook outcome: a composite measure for quality of elective aneurysm surgery. Ann Surg. 2017;266:898-904.

14. Busweiler LA, Schouwenburg MG, van Berge Henegouwen MI, et al. Textbook outcome as a composite measure in oesophagogastric cancer surgery. $\mathrm{Br}$ J Surg. 2017;104:742-750.

15. Kolfschoten NE, Kievit J, Gooiker GA, et al. Focusing on desired outcomes of care after colon cancer resections; hospital variations in 'textbook outcome'. Eur J Surg Oncol. 2013;39:156-163.

16. Mull HJ, Chen Q, Shwartz M, et al. Measuring surgical quality: which measure should we trust? JAMA Surg. 2014;149:1210-1212.

17. van der Kaaij RT, de Rooij MV, van Coevorden F, et al. Using textbook outcome as a measure of quality of care in oesophagogastric cancer surgery. $\mathrm{Br}$ J Surg. 2018;105:561-569.

18. Salet N, Bremmer RH, Verhagen M, et al. Is textbook outcome a valuable composite measure for short-term outcomes of gastrointestinal treatments in the Netherlands using hospital information system data? A retrospective cohort study. BMJ Open. 2018;8:e019405.

19. Inzani F, Petrone G, Rindi G. The New World Health Organization Classification for Pancreatic Neuroendocrine Neoplasia. Endocrinol Metab Clin North Am. 2018;47:463-470.

20. Bassi C, Marchegiani G, Dervenis C, et al. The 2016 update of the International Study Group (ISGPS) definition and grading of postoperative pancreatic fistula: 11 years after. Surgery. 2017;161:584-591.

21. Wente MN, Veit JA, Bassi C, et al. Postpancreatectomy hemorrhage (PPH): an International Study Group of Pancreatic Surgery (ISGPS) definition. Surgery. 2007;142:20-25.

22. Koch M, Garden OJ, Padbury R, et al. Bile leakage after hepatobiliary and pancreatic surgery: a definition and grading of severity by the International Study Group of Liver Surgery. Surgery. 2011;149:680-688.

23. Dindo D, Demartines N, Clavien PA. Classification of surgical complications: a new proposal with evaluation in a cohort of 6336 patients and results of a survey. Ann Surg. 2004;240:205-213.

24. Kristensen SR, Bech M, Quentin W. A roadmap for comparing readmission policies with application to Denmark, England, Germany and the United States. Health Policy. 2015;119:264-273.
25. Fischer C, Lingsma HF, Marang-van de Mheen PJ, et al. Is the readmission rate a valid quality indicator? A review of the evidence. PLoS One. 2014;9:e112282.

26. Merath K, Chen Q, Bagante F, et al. Textbook outcomes among medicare patients undergoing hepatopancreatic surgery. Ann Surg. 2018. Nov 29. doi: 10.1097/SLA.0000000000003105. [Epub ahead of print].

27. Verbeke CS, Menon KV. Redefining resection margin status in pancreatic cancer. HPB (Oxford). 2009;11:282-289.

28. Merkow RP, Bilimoria KY, Bentrem DJ, et al. National assessment of margin status as a quality indicator after pancreatic cancer surgery. Ann Surg Oncol. 2014;21:1067-1074.

29. Maggino L, Liu JB, Ecker BL, et al. Impact of operative time on outcomes after pancreatic resection: a risk-adjusted analysis using the American College of Surgeons NSQIP Database. J Am Coll Surg. 2018;226:844.e3-857.e3.

30. Ball CG, Pitt HA, Kilbane ME, et al. Peri-operative blood transfusion and operative time are quality indicators for pancreatoduodenectomy. $H P B$ (Oxford). 2010;12:465-471.

31. McMillan MT, Allegrini V, Asbun HJ, et al. Incorporation of procedurespecific risk into the ACS-NSQIP surgical risk calculator improves the prediction of morbidity and mortality after pancreatoduodenectomy. Ann Surg. 2017;265:978-986.

32. Callery MP, Pratt WB, Kent TS, et al. A prospectively validated clinical risk score accurately predicts pancreatic fistula after pancreatoduodenectomy. $J$ Am Coll Surg. 2013;216:1-14.

33. Braga M, Capretti G, Pecorelli N, et al. A prognostic score to predict major complications after pancreaticoduodenectomy. Ann Surg. 2011;254: $702-707$.

34. Ecker BL, McMillan MT, Allegrini V, et al. Risk factors and mitigation strategies for pancreatic fistula after distal pancreatectomy: analysis of 2026 resections from the International, Multi-institutional Distal Pancreatectomy Study Group. Ann Surg. 2019;269:143-149.

35. Peng YP, Zhu XL, Yin LD, et al. Risk factors of postoperative pancreatic fistula in patients after distal pancreatectomy: a systematic review and metaanalysis. Sci Rep. 2017;7:185.

36. van Rijssen LB, Zwart MJ, van Dieren S, et al. Variation in hospital mortality after pancreatoduodenectomy is related to failure to rescue rather than major complications: a nationwide audit. HPB (Oxford). 2018;20:759-767. 